\title{
Rosenbrock methods for solving Riccati differential equations
}

\author{
Peter Benner Hermann Mena
}

\begin{abstract}
The Riccati differential equation (RDE) arises in several fields like optimal control, optimal filtering, $\mathrm{H}_{\infty}$ control of linear timevarying systems, differential games, etc. In the literature there is a large variety of approaches to compute its solution. Particularly for stiff RDEs, matrix-valued versions of the standard multi-step methods for solving ordinary differential equations have given good results. In this paper we discuss a particular class of one-step methods. These are the linearimplicit Runge-Kutta methods or Rosenbrock methods. We show that they offer a practical alternative for solving stiff RDEs. They can be implemented with good stability properties and allow for a cheap step size control. The matrix valued version of the Rosenbrock methods for RDEs requires the solution of one Sylvester equation in each stage of the method. For the case in which the coefficient matrices of the Sylvester equation are dense, the Bartels-Stewart method can be efficiently applied for solving the equations. The computational cost (computing time and memory requirements) is smaller than for the multi-step methods.
\end{abstract}

Index Terms-Riccati differential equation, Rosenbrock methods, linear-implicit Runge-Kutta, Sylvester equation

\section{INTRODUCTION}

We consider time-varying Riccati differential equations (RDEs) of the form

$$
\begin{aligned}
\dot{X}(t) & =Q(t)+X(t) A(t)+B(t) X(t)-X(t) R(t) X(t) \\
& \equiv F(t, X(t)), \\
X\left(t_{0}\right) & =X_{0},
\end{aligned}
$$

where $t \in\left[t_{0}, t_{f}\right], A(t) \in \mathbb{R}^{n \times n}, B(t) \in \mathbb{R}^{m \times m}, Q(t) \in \mathbb{R}^{m \times n}$, $R(t) \in \mathbb{R}^{n \times m}, X(t) \in \mathbb{R}^{m \times n}$. We assume that the coefficient matrices are piecewise continuous locally bounded matrix-valued functions, which ensure the existence and uniqueness of the solution of (1), see, e.g., [1, Thm. 4.1.6]. The RDE is called symmetric, if $Q(t), R(t)$ are square, symmetric matrices and $B(t)=A(t)^{T}$ for all $t \in\left[t_{0}, t_{f}\right]$.

Symmetric RDEs arise from linear-quadratic optimal control problems like LQR and LQG design with finite-time horizon, in $H_{\infty}$ control of linear-time varying systems as well as in differential games; see, e.g., [1], [2]. Unfortunately, in most control problems fast and slow modes are present. This implies that the associated RDE will be fairly stiff, which in turn demands for implicit methods to solve such RDEs numerically. Therefore, we will focus here on the stiff case. In this context, several approaches to solve RDEs have been proposed, [3], [4], [5]. Particularly, matrix-valued algorithms for solving RDEs based on generalizations of the BDF methods have been considered, see [6], [7], [8]. These methods are also suitable for large scale RDEs arising in optimal control problems for parabolic partial differential equations [9].

In general, the BDF methods require fewer function evaluations per step than one step methods, and they allow a simpler streamlined method design from the point of view of order and error estimation. However, the associated overhead is higher for changing the step size compared with one step methods. Diagonally implicit RungeKutta methods or collocation methods offer an alternative to the BDF methods for solving stiff problems. Especially, linearly implicit onestep methods (better known as Rosenbrock methods) give satisfactory

Max Planck Institute for Dynamics of Complex Technical Systems, 39106 Magdeburg, and TU Chemnitz, Department of Mathematics, Germany benner@mpi-magdeburg.mpg.de

University of Innsbruck, Department of Mathematics, A-6020 Innsbruck, Austria, hermann.mena@uibk.ac.at results see, e.g., [10], [11]. The idea of these methods can be interpreted as the application of one Newton iteration to each stage of an implicit Runge-Kutta method and the derivation of stable formulae by working with the Jacobian matrix directly within the integration formulae. Here, we derive a matrix valued version of the Rosenbrock methods for solving RDEs.

In the literature, variants of the Rosenbrock method are discussed, in which the Jacobian matrix is retained over several steps or even replaced by an approximation which renders the linear system cheaper. Methods constructed in this way were first studied by T. Steihaug and A. Wolfbrand in 1979. Since they denoted the inexact Jacobi matrix by " $W$ ", these methods are often called $W$-methods. In general, they are a practical alternative when low accuracy is prescribed. The application of these ideas to the matrix-valued Rosenbrock methods discussed is straight forward.

This paper is organized as following. First, we will review matrixvalued versions of numerical methods for RDEs. Then, in Section II, we describe the Rosenbrock methods, discuss stability properties and show some particular schemes. The application of this method to RDEs is shown in Section III, as well as implementation details. In Section IV numerical experiments are discussed. The paper is concluded by a brief summary and outlook on future work.

\section{ROSENBROCK METHODS}

As described in [7], the numerical methods for solving RDEs of the form (1) can essentially be distinguished into five classes: unroll the matrices into vectors and integrate the resulting system, linearize the RDE and solve the resulting Hamiltonian type system, transform the RDE into two coupled nonlinear equations (the socalled Chandrasekhar system), methods based on the superposition property of the Riccati solutions and matrix versions of the ODE methods. The latter is, in general, the most efficient method. We refer the reader to [7], [8] and references therein for a detailed explanation. Moreover, as we consider stiff RDEs the BDF methods are the natural choice. These methods solve the RDE using matrixvalued algorithms based on standard BDF methods for solving ODEs [7], [8]. The application of the BDF methods yields an algebraic Riccati equation (ARE) to be solved in each time step. Since the ARE is a nonlinear matrix equation, it is natural to apply Newton's method or variants of it. The application of Newton's method yields a Sylvester equation to be solved in every step, details and further references can be found in [12].

Other matrix-valued algorithms yield an ARE to be solved in every step also, e.g., the Midpoint or Trapezodial rules. In fact, as stated in [8], mainly every implicit scheme yields an ARE to be solved in every step. Note that in infinite arithmetics solving a vectorized version of the RDE by a standard ODE method will produce the same solution as the one obtained using the matrix-value version of this ODE method [8]. For stiff RDEs the BDF methods are a good choice among multistep methods [7]. In the following we will see that Rosenbrock methods offer a practical alternative to the BDFs methods.

The general $s$-stage Rosenbrock method for a non-autonomous ODE system

$$
\dot{x}=f(t, x), \quad x\left(t_{0}\right)=x_{0},
$$


with $f:\left[t_{0}, t_{f}\right] \times \mathbb{R}^{n} \rightarrow \mathbb{R}^{n}$ bounded in the neighborhood of $x_{0}$ and Lipschitz continuous in $x$, is given as follows: for $i=1, \ldots, s$,

$$
\begin{array}{rl}
k_{i}=h & h\left(t_{k}+\alpha_{i} h, x_{k}+\sum_{j=1}^{i-1} \alpha_{i j} k_{j}\right) \\
& +\gamma_{i} h^{2} \frac{\partial f}{\partial t}\left(t_{k}, x_{k}\right)+h \frac{\partial f}{\partial x}\left(t_{k}, x_{k}\right) \sum_{j=1}^{i} \gamma_{i j} k_{j}, \\
x_{k+1}= & x_{k}+\sum_{j=1}^{s} b_{j} k_{j}
\end{array}
$$

where $\alpha_{i j}, \gamma_{i j}, b_{j}$ are the determining coefficients and $h$ is the step size. The additional coefficients are given by

$$
\alpha_{i}=\sum_{j=1}^{i-1} \alpha_{i j}, \quad \gamma_{i}=\sum_{j=1}^{i} \gamma_{i j} .
$$

Each stage of this method consists of a system of linear equations with unknowns $k_{i}$. Note that for the autonomous case, i.e., $f(t, x) \equiv f(x)$, the coefficients from (4) do not appear and therefore the iteration is simplified. Also of special interest are the methods for which $\gamma_{11}=\cdots=\gamma_{s s}=\gamma$, for which A-stability and L-stability can be achieved, see, e.g. [11, Section IV.7]. The order of the resulting methods is $s$ or $s+1$ for specific values of $\gamma$. That is because in general Rosenbrock methods have the same stability function as diagonally implicit Runge-Kutta methods, [11]. Rosenbrock methods are very attractive for several reasons like: excellent stability properties, easy to implement, cheap step size control, suitability for parallelization, applicability to implicit systems of the form $M \dot{y}=f(y)$, among others. In order to avoid matrix-vector multiplications, in the implementation of the method new variables $u_{i}=\sum_{j=1}^{i} \gamma_{i j} k_{j}$ are introduced. Thus, the $s$-stage Rosenbrock method (3) can be written as

$$
\begin{aligned}
\left(\frac{1}{h \gamma_{i i}} I-\frac{\partial f}{\partial x}\left(t_{k}, x_{k}\right)\right) u_{i}= & f\left(t_{k}+\alpha_{i} h, x_{k}+\sum_{j=1}^{i-1} a_{i j} u_{j}\right) \\
& +\sum_{j=1}^{i-1} \frac{c_{i j}}{h} u_{j}+\gamma_{i} h \frac{\partial f}{\partial t}\left(t_{k}, x_{k}\right) \\
x_{k+1}= & x_{k}+\sum_{j=1}^{s} m_{j} u_{j}
\end{aligned}
$$

where $i=1, \ldots, s$, and using $\Gamma=\left(\gamma_{i j}\right)$,

$$
\begin{aligned}
C & =\operatorname{diag}\left(\gamma_{11}^{-1}, \ldots, \gamma_{s s}^{-1}\right)-\Gamma^{-1} \\
\left(a_{i j}\right) & =\left(\alpha_{i j}\right) \Gamma^{-1} \\
\left(m_{1}, \ldots, m_{s}\right) & =\left(b_{1}, \ldots, b_{s}\right) \Gamma^{-1}
\end{aligned}
$$

The Rosenbrock methods have been deeply studied, e.g., in [11].

\section{A. Rosenbrock schemes}

a) Linear-implicit Euler method: The one stage Rosenbrock method, also known as the linear-implicit Euler method, for nonautonomous systems becomes

$$
\begin{aligned}
\left(I-\frac{\partial f}{\partial x}\left(t_{k}, x_{k}\right) h\right) k_{1} & =f\left(x_{k}\right)+h \frac{\partial f}{\partial t}\left(t_{k}, x_{k}\right), \\
x_{k+1} & =x_{k}+h k_{1} .
\end{aligned}
$$

The coefficients are chosen as $b_{1}=1, \gamma=1$ and $\alpha_{11}=0$. The method is of order $p=1$ and the stability function is the same as the one for the implicit Euler method. b) A second order method: In [13] a second order method for autonomous ODE systems is applied to atmospheric dispersion problems describing photochemistry, advective, and turbulent diffusive transport. For non-autonomous systems the scheme can be written as

$$
\begin{aligned}
\left(I-\gamma h \frac{\partial f}{\partial x}\left(t_{k}, x_{k}\right)\right) k_{1}= & f\left(t_{k}, x_{k}\right)+\gamma h \frac{\partial f}{\partial t}\left(t_{k}, x_{k}\right), \\
\left(I-\gamma h \frac{\partial f}{\partial x}\left(t_{k}, x_{k}\right)\right) k_{2}= & f\left(t_{k}+h, x_{k}+h k_{1}\right)-2 k_{1} \\
& -\gamma h \frac{\partial f}{\partial t}\left(t_{k}, x_{k}\right), \\
x_{k+1}= & x_{k}+\frac{h}{2}\left(3 k_{1}+k_{2}\right) .
\end{aligned}
$$

It is pointed out by the authors that the method is capable of integrating with large a priori described step sizes for the problem that they are dealing with [13].

Te methods described above have already proven to be very effective in many applications, e.g. see [13], [14]. Most of the computational cost is related with the evaluation of the partial derivatives of $f$ at every step. In general the latter is considered expensive. In the next section we explain how to efficiently implement this for solving RDEs.

\section{ApplicAtion to RDEs}

We consider time varying RDEs of the form (1). The application of the Rosenbrock method (5), as a matrix-valued algorithm, to the RDE (1) yields

$$
\begin{aligned}
\left(\frac{1}{h \gamma_{i i}} I-\frac{\partial F}{\partial X}\left(t_{k}, X_{k}\right)\right) K_{i}= & F\left(t_{k, i}, X_{k}+\sum_{j=1}^{i-1} a_{i j} K_{j}\right) \\
& +\sum_{j=1}^{i-1} \frac{c_{i j}}{h} K_{j}+\gamma_{i} h F_{t_{k}} \\
X_{k+1}= & X_{k}+\sum_{j=1}^{s} m_{j} K_{j},
\end{aligned}
$$

where $t_{k, i}=t_{k}+\alpha_{i} h, i=1, \ldots, s$, and the coefficients of the method are defined as in (4) and (6). We use $K_{i}$ instead of $u_{i}$, note that $K_{i}$ represent $n \times n$ matrix. We also note $F_{t_{k}}=\frac{\partial F}{\partial t}\left(t_{k}, X\left(t_{k}\right)\right)$. The derivative $\frac{\partial F}{\partial X}\left(t_{k}, X_{k}\right)$ in (9) is given by the (Frechét) derivative of $F$ at $X_{k}$, represented by the Sylvester operator

$$
\frac{\partial F}{\partial X}\left(t_{k}, X_{k}\right): U \rightarrow\left(B_{k}^{T}-R_{k} X_{k}\right)^{T} U+U\left(A_{k}-R_{k} X_{k}\right),
$$

where $X_{k} \approx X\left(t_{k}\right), A_{k} \equiv A\left(t_{k}\right), B_{k} \equiv B\left(t_{k}\right), R_{k} \equiv R\left(t_{k}\right)$ and $U \in \mathbb{R}^{n \times n}$.

Replacing $\frac{\partial F}{\partial X}\left(t_{k}, X_{k}\right)$ in the left hand side of the first equation in (9) we obtain,

$$
\frac{1}{h \gamma_{i i}} K_{i}-\left(B_{k}^{T}-R_{k} X_{k}\right)^{T} K_{i}-K_{i}\left(A_{k}-R_{k} X_{k}\right),
$$

and re-arranging terms yields

$-\left(\left(B_{k}^{T}-R_{k} X_{k}-\frac{1}{2 h \gamma_{i i}} I\right)^{T} K_{i}+K_{i}\left(A_{k}-R_{k} X_{k}-\frac{1}{2 h \gamma_{i i}} I\right)\right)$.

Defining

$$
\begin{aligned}
\bar{A}_{k} & =A_{k}-R_{k} X_{k}-\frac{1}{2 h \gamma_{i i}} I \\
\bar{B}_{k} & =\left(B_{k}^{T}-R_{k} X_{k}-\frac{1}{2 h \gamma_{i i}} I\right)^{T},
\end{aligned}
$$


we can write (9) for $i=1, \ldots, s$ as

$$
\begin{aligned}
\bar{B}_{k} K_{i}+K_{i} \bar{A}_{k}= & -F\left(t_{k, i}, X_{k}+\sum_{j=1}^{i-1} a_{i j} K_{j}\right) \\
& -\sum_{j=1}^{i-1} \frac{c_{i j}}{h} K_{j}-\gamma_{i} h F_{t_{k}}, \\
X_{k+1}= & X_{k}+\sum_{j=1}^{s} m_{j} K_{j},
\end{aligned}
$$

Hence, one Sylvester equation has to be solved in each stage of the method every time step. But as the coefficient matrices $\bar{A}_{k}, \bar{B}_{k}$ are the same for all stages, an initial Schur or Hessenberg-Schur reduction as proposed in [15], [16] can be re-used in all stages so that the main cost is proportional to one Sylvester solve. This can be exploited using, e.g., the Sylvester solver from SLICOT ${ }^{1}$, which is accessible in MATLAB as function slsylv provided by the SLICOT Basic Systems and Control Toolbox ${ }^{2}$. This property can be used to efficiently implement the method, especially if the dimension of the problem is large, i.e., the size of the matrix $X$. Note that for the matrix valued BDF methods applied to RDEs, one ARE has to be solved every time step [17], [7]. There are many efficient methods available in the literature for solving AREs, see, e.g., [18]. A common approach for the type of RDEs we considered is using Newton's method. Thus, a Sylvester equation with changing coefficient matrices has to be solved in every Newton iteration; usually several iterations are required to achieve the desired precision [12]. Hence, the computational cost for solving RDEs using the Rosenbrock methods is reduced by a factor of $k$, where $k$ is the average number of Newton steps, compared with the one using the BDF methods. In a number of numerical experiments provided in [9], Newton's method typically converges in 3-5 iterations in this context. Alternatively, the Schur method [19] can be applied to solve AREs. This algorithm requires $205 n^{3}$ flops which is roughly more than three times the amount of flops required for solving a Sylvester equation [18]. In this case the computational cost is reduced roughly by a factor of three. For the symmetric case, a comparison of implementations based on the Bartels-Stewart method [15] for the Lyapunov equation, coming at a cost of $32 n^{3}$ flops, and the Schur vector method for the ARE leads to basically a speed-up of factor 5-6 per time step, counting floating point operations.

Note that working with an $s$-stage Rosenbrock method, the intermediate approximation at stage $s-1$ can be used to cheaply estimate the local error for controling the step size. For instance, the intermediate approximation of the second order Rosenbrock method (8) corresponds to the application of the linear-implicit Euler method at $t_{k+1}$. Taking this into account, a general implementation of the $s$-stage Rosenbrock method using step size control is sketched in Algorithm III.1. There, the growth of the step is limited by a factor $q>1$ and the maximum step size allowed $h_{\max }$.

\section{A. Implementation details}

If $F\left(t_{k}+\alpha_{i} h, X_{k}+\sum_{j=1}^{i-1} a_{i j} K_{j}\right)$ in (10) is written as

$$
\begin{aligned}
\left(B_{k_{h}}^{T}\right. & \left.-R_{k_{h}} \bar{X}_{k}-\frac{1}{2 h \gamma_{i i}} I\right)^{T} \bar{X}_{k} \\
& +\bar{X}_{k}\left(A_{k_{h}}-R_{k_{h}} \bar{X}_{k}-\frac{1}{2 h \gamma_{i i}} I\right) \\
& +Q_{k_{h}}+\bar{X}_{k_{h}} R_{k_{h}} \bar{X}_{k}+\frac{1}{h \gamma_{i i}} \bar{X}_{k}
\end{aligned}
$$

\footnotetext{
${ }^{1}$ Subroutine Library in Control, available at www.slicot.org

${ }^{2}$ See http://www.slicot.org/index.php?site=slbasic
}

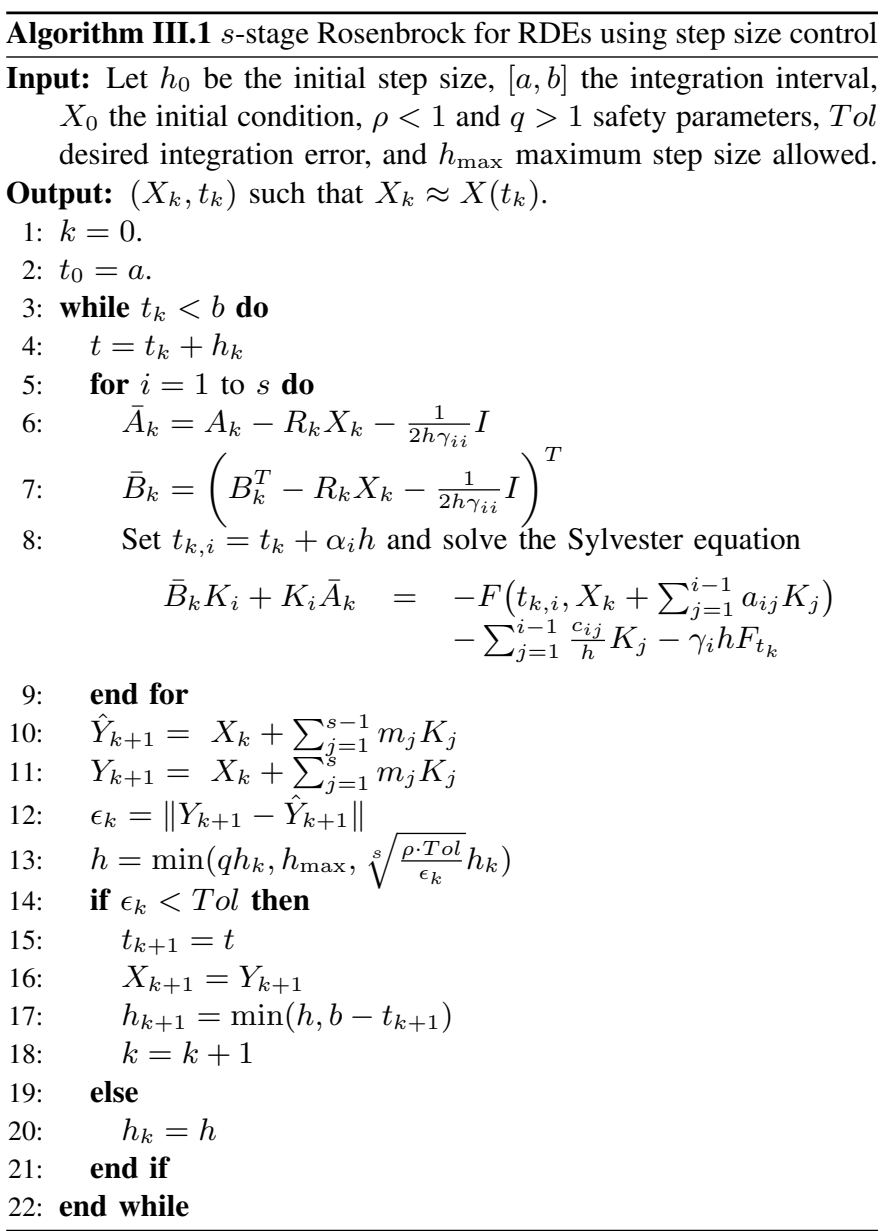

where $A_{k_{h}}=A\left(t_{k}+\alpha_{i} h\right), B_{k_{h}}=B\left(t_{k}+\alpha_{i} h\right), R_{k_{h}}=R\left(t_{k}+\alpha_{i} h\right)$ and $\bar{X}_{k}=X_{k}+\sum_{j=1}^{i-1} a_{i j} K_{j}$, then, in some cases we can simplify the implementation of the method.

Let us consider the linear-implicit Euler method applied to RDEs

$$
\begin{aligned}
\bar{B}_{k} K_{1}+K_{1} \bar{A}_{k} & =-F\left(X_{k}\right)-h F_{t_{k}}, \\
X_{k+1} & =X_{k}+K_{1}
\end{aligned}
$$

where

$$
\begin{aligned}
& \bar{A}_{k}=A_{k}-R_{k} X_{k}-\frac{1}{2 h} I \\
& \bar{B}_{k}=\left(B_{k}^{T}-R_{k} X_{k}-\frac{1}{2 h} I\right)^{T} .
\end{aligned}
$$

If we write $F\left(X_{k}\right)$ analogously to (11), then we can re-write (12) such that the next iterate is computed directly from the Sylvester equation,

$$
\bar{B}_{k} X_{k+1}+X_{k+1} \bar{A}_{k}=-Q_{k}-X_{k} R_{k} X_{k}-\frac{1}{h} X_{k}-h F_{t_{k}} .
$$

The right hand side of (13) is simpler to evaluate than the one in (12), so the implementation of (13) is more efficient.

Let us now consider the second-order Rosenbrock method (8), as a matrix-valued algorithm, applied to the RDE (1)

$$
\begin{aligned}
\bar{B}_{k} K_{1}+K_{1} \bar{A}_{k} & =-F\left(t_{k+1}, X_{k}\right)-h \gamma F_{t_{k}} \\
\bar{B}_{k} K_{2}+K_{2} \bar{A}_{k} & =-F\left(t_{k+1}, X_{k}+h K_{1}\right)+2 K_{1}+h \gamma F_{t_{k}} \\
X_{k+1} & =X_{k}+\frac{3}{2} K_{1}+\frac{1}{2} K_{2}
\end{aligned}
$$


where $t_{k+1}=t_{k}+h$ and

$$
\begin{aligned}
& \bar{A}_{k}=A_{k}-R_{k} X_{k}-\frac{1}{2 h \gamma} I, \\
& \bar{B}_{k}=\left(B_{k}^{T}-R_{k} X_{k}-\frac{1}{2 h \gamma} I\right)^{T},
\end{aligned}
$$

Rewriting the right hand side of the second Sylvester equation in (14) analogously to (11) as

$$
\begin{aligned}
& -F\left(t_{k+1}, X_{k}\right)+\frac{1}{h \gamma} K_{1}+\gamma h F_{t_{k}}-h^{2} K_{1} R_{k+1} K_{1}+2 K_{1} \\
& -\left(B_{k+1}^{T}-R_{k+1} X_{k}-\frac{1}{2 h \gamma} I\right)^{T} K_{1} \\
& -K_{1}\left(A_{k+1}-R_{k+1} X_{k}-\frac{1}{2 h \gamma} I\right)
\end{aligned}
$$

under the assumption that the size of the matrix $X$ is large and due to the linearity of the Sylvester equation, it is more efficient to solve an additional equation (with the same coefficient matrices $\bar{A}_{k}$ and $\bar{B}_{k}$ ) than solving (14) directly. The right hand side of this equation is chosen as the common factor of the right hand sides of the equations involved in each stage. Then, the original solution is recovered afterwards. The standard implementation of this second order Rosenbrock method is sketched in Algorithm III.2, which can be endowed with step size control as in Algorithm III.1.

Again, note that the additional solve is cheap if we exploit the decompositions of $\bar{A}_{K}, \bar{B}_{k}$ and only perform the backward substitution step of the Hessenberg-Schur or Bartels-Stewart methods. Finally, we point out that a similar implementation can be straightforward performed for any $s$-stage Ronsenbrock method.

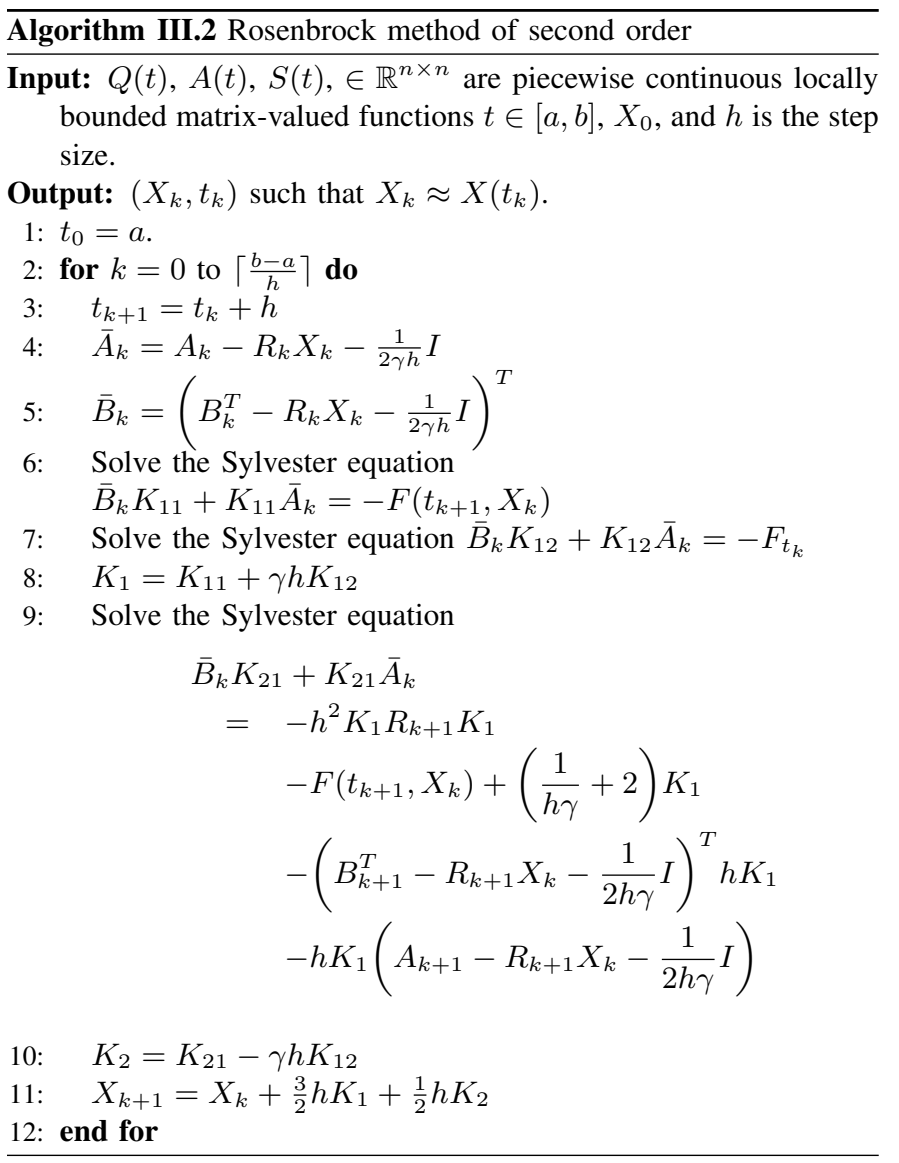

\section{B. Autonomous RDE}

For autonomous RDEs, i.e., RDEs, in which the coefficients matrices $Q(t), A(t), R(t)$ are constant, $F\left(t_{k+1}, X_{k}\right) \equiv F\left(X_{k}\right)$, so $F_{t_{k}}=0$. Particularly, for the second order Rosenbrock method this term, arising in the second Sylvester equation,

$$
-\left(B^{T}-R X_{k}-\frac{1}{2 h \gamma} I\right)^{T} h K_{1}-h K_{1}\left(A-R X_{k}-\frac{1}{2 h \gamma} I\right)
$$

is equal to $h F\left(X_{k}\right)$ because of the linearity of the solution. Therefore, the method can be written like:

$$
\begin{aligned}
X_{k+1}= & X_{k}+\frac{3}{2} h K_{1}+\frac{1}{2} h K_{2}, \\
\hat{B}_{k} K_{1}+K_{1} \hat{A}_{k}= & -F\left(X_{k}\right), \\
\hat{B}_{k} K_{2}+K_{2} \hat{A}_{k}= & -h^{2} K_{1} R K_{1}+\left(\frac{1}{h \gamma}+2\right) K_{1} \\
& +(h-1) F\left(X_{k}\right),
\end{aligned}
$$

where $\hat{A}_{k}=A-R X_{k}-\frac{1}{2 h \gamma} I, \hat{B}_{k}=\left(B^{T}-R X_{k}-\frac{1}{2 h \gamma} I\right)^{T}$. Moreover, it can be efficiently computed as:

$$
\begin{aligned}
X_{k+1} & =X_{k}+\frac{3}{2} h K_{1}+\frac{1}{2} h K_{2}, \\
\hat{B}_{k} K_{1}+K_{1} \hat{A}_{k} & =-F\left(X_{k}\right), \\
\hat{B}_{k} K_{21}+K_{21} \hat{A}_{k} & =-h^{2} K_{1} R K_{1}+\left(\frac{1}{h \gamma}+2\right) K_{1}, \\
K_{2} & =K_{21}-(h-1) K_{1} .
\end{aligned}
$$

In control theory, particularly solving finite dimensional linear quadratic control problems we have to deal with symmetric RDEs. Thus, instead of a Sylvester equation, the solution of a Lyapunov equation in every stage of a Rosenbrock method is required. Particularly, for large scale RDEs arising from the discretization in space of the optimal control problems governed by partial differential equations of parabolic type an efficient implementation of the Rosenbrock methods is proposed in [9]. The key ingredient there is to find a low rank approximation of the solution and to rewrite the method for the these low rank factors of the solution.

The ideas described here can be in principle be applied to any method including the popular Rosenbrock methods of order four, [20].

\section{NUMERICAL RESULTS}

In the following, we will demonstrate the performance of the proposed algorithms for two typical RDE test examples from the literature. Due to space limitations, we restrict ourselves to this limited number of tests. A detailed description of the implementation details and performance results for different hardware platforms will be reported elsewhere. All tests were performed on a standard desktop computer running MATLAB ${ }^{\circledR}$ Version 2012a using IEEE double precision arithmetic. We have used implementations of Algorithm III.1 for $s=1$ (linear-implicit Euler, Ros1) and $s=2$ (Ros2), the implementation for the latter follows the description given in Algorithm III.2. In order to demonstrate the efficiency of a practical variant that might serve as standard solver for RDEs, if implemented professionally in a standardized way, we have combined the 1- and 2-stage Rosenbrock methods to a variable step-size and order method Ros 12. Here, the step-size control is implemented as described in Algorithm III. 1 with $\rho=0.9, q=1.5, h_{\max }=0.1$, and a problem-dependent tolerance $\mathrm{Tol}$. The variable-order component of the algorithm follows [10, Section 7.4.1]. We compare this method for one example with BDF123, which is an implementation of the BDF methods of orders 1,2 , and 3 with variable step size and order control (again following [10, Section 7.4.1]). By this, we demonstrate 


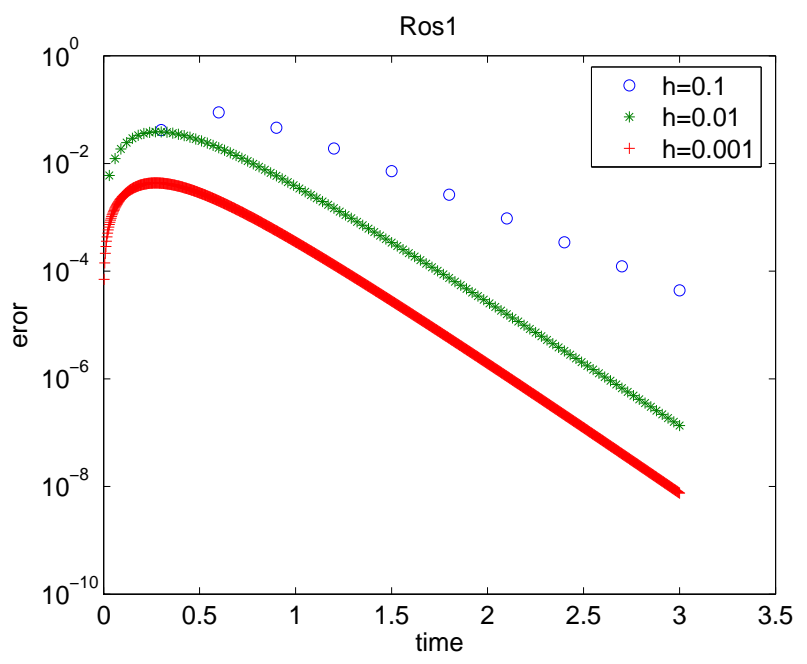

(a)

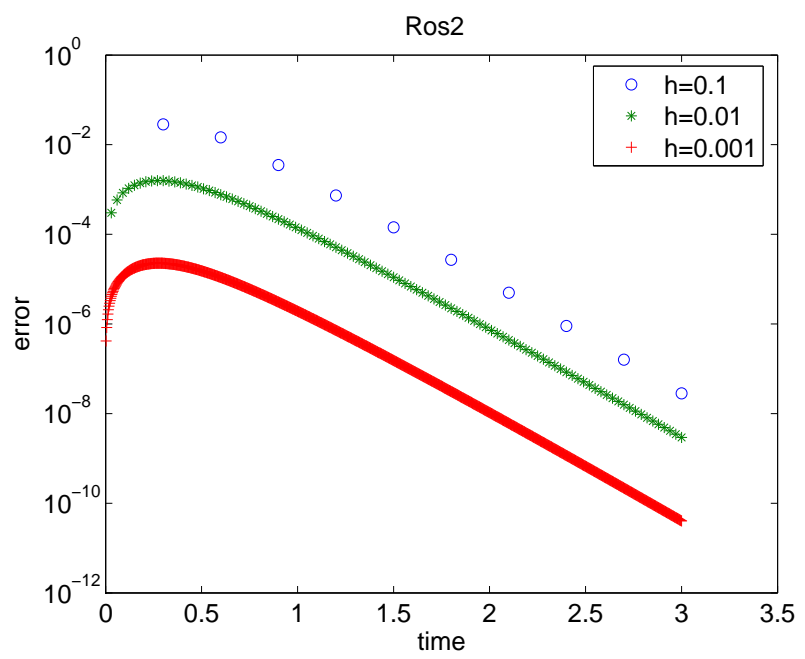

(b)

Fig. 1. Example 1 (interval of integration is $[0,3]$ ) (a) error per step using fixed step sizes $h=0.1, h=0.01$, and $h=0.001$ by the linearly implicit Euler method (Ros1) and by the Rosenbrock method of order two (Ros2) (b)

that Ros12 can achieve the same or better accuracy as BDF123 with similar or larger time steps, resulting in a much reduced cost for Ros 12 compared to BDF123 as only 1 or 3 Sylvester/Lyapunov equations have to be solved per time step compared to one ARE being the main cost of the BDF method as already explained in Section III. For an explicit comparison of CPU times, we again have to refer to future work.

\section{A. Example 1}

Let us now consider the following symmetric RDE of size $n$,

$$
\begin{aligned}
& \dot{X}(t)=-X^{2}(t)+k^{2} I_{n}, \quad t_{0} \leq t \leq T \\
& X\left(t_{0}\right)=X_{0} .
\end{aligned}
$$

If $X_{0}$ is diagonalizable, i.e, $X_{0}=S \Lambda S^{-1}$ with $\Lambda=\operatorname{diag}\left[\lambda_{i}\right]$, then the analytic solution of (19) is:

$$
X(t)=S \operatorname{diag}\left[\frac{k \sinh k t+\lambda_{i} \cosh k t}{\cosh k t+\frac{\lambda_{i}}{k} \sinh k t}\right] S^{-1},
$$

refer to [21] for a detailed explanation. Here, we choose

$$
X_{0}=I_{n}, \quad k=3, \quad n=60 .
$$

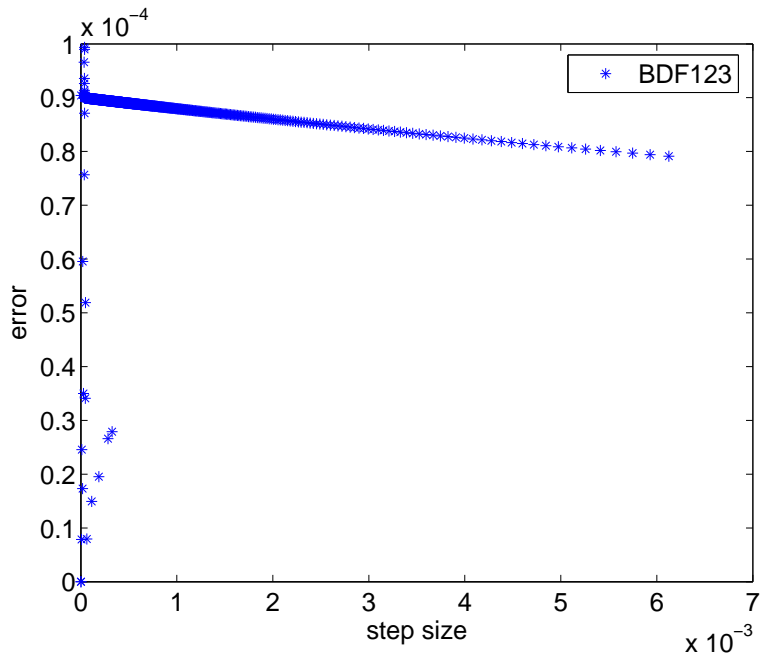

(a)

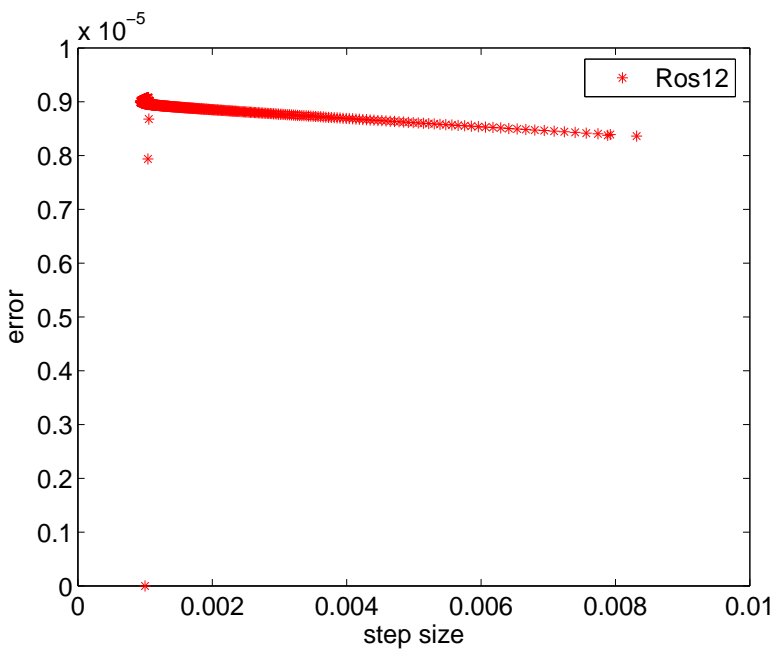

(b)

Fig. 2. Example 1 (a) error vs. step size by the variable step size and order BDF method up to order 3 (BDF123), (b) error vs. step size y the variable step size Rosenbrock method of order two (Ros12)

In Figure 1 (a) the error per step using fixed step sizes $h=0.1$, $h=0.01$, and $h=0.001$ by the linearly implicit Euler method (Ros1) and by the Rosenbrock method of order two (b) (Ros2) is plotted. We can observe that the order of the method is achieved. In Figure 2 the error vs. step size by a variable step size/order method is shown in (a) for the BDF methods up to order three (BDF123) and in (b) for the Rosenbrock method of up to order two (Ros12). The tolerance to accept or redo the current step was choosen as $\mathrm{Tol}=$ $1 e-4$. We can observe that the performance of the Rosenbrock of order two is competitive achieving higher precision with essentially the same step size at a lower computational cost.

\section{B. Example 2}

This example was taken from the SLICOT collection of benchmark examples for continuous-time algebraic Riccati equations [22, Example 4.2]. Here, we consider the associated RDE

$$
\begin{aligned}
\dot{X}(t)= & \left(C^{T} \tilde{Q}\right)\left(C^{T} \tilde{Q}\right)^{T}+A^{T} X(t)+X(t) A \\
& -X(t)\left(B \tilde{R}^{-1} \tilde{R}\right)\left(B \tilde{R}^{-1} \tilde{R}\right)^{T} X(t),
\end{aligned}
$$




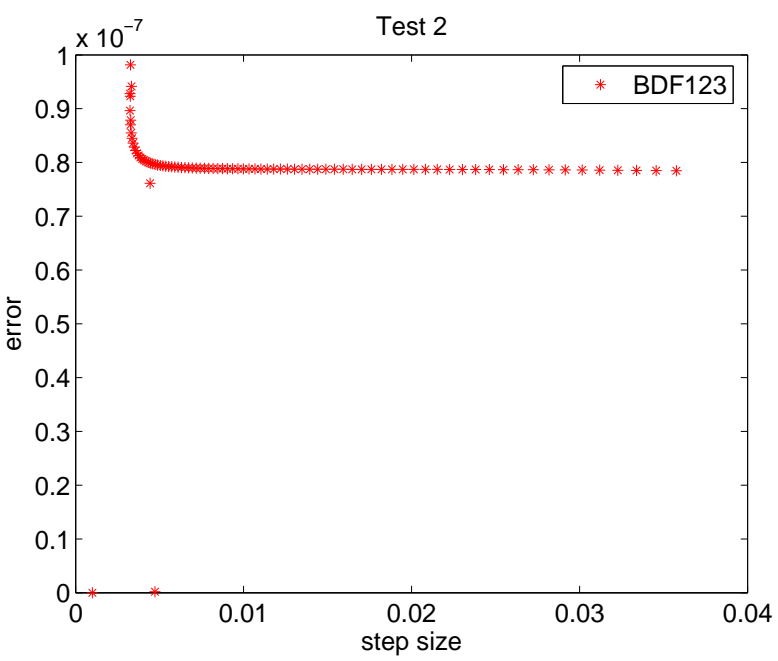

(a)

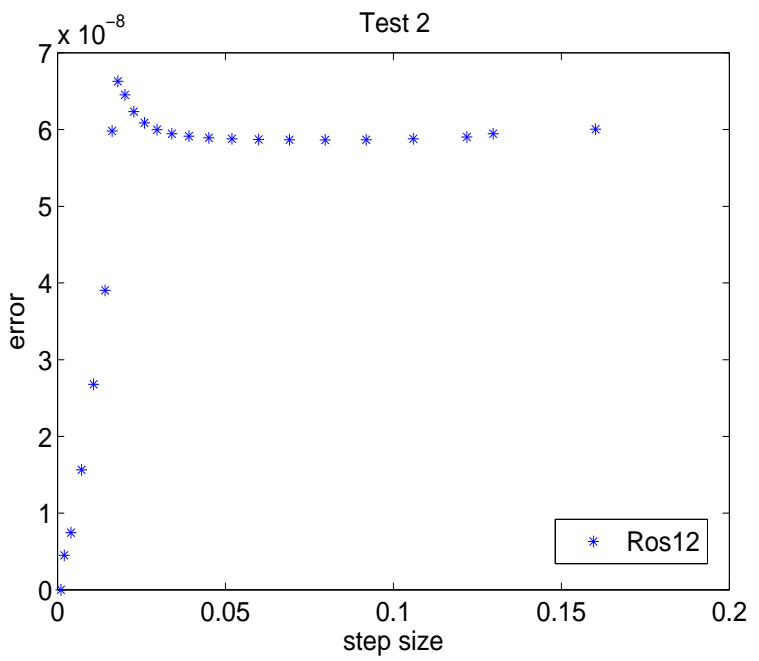

(b)

Fig. 3. Example 2, interval of integration $[0,1], T o l=1 e-7$ (a) error vs. step size for BDF123 (b) error vs. step size for Ros 12 .

where the matrices $C, \tilde{Q}, A, B$ and $\tilde{R}$ come from the ARE arising in the discretized problem. The initial condition for (20) is equal to zero. The problem parameters are set as the default values of this benchmark example and dimension of the system as $n=100$, [22, Example 4.2].

In Figure 3 the error vs. step size by BDF123 is shown in (a). The same data are plotted for Ros12 in (b), respectively. The tolerance for accept or redo the current step was choosen as $T o l=1 e-7$. As in the previous example we observe that the Rosenbrock methods achieve higher precision than BDF methods even with much fewer steps. Recall that in Rosenbrock methods, only Lyapunov equations need to be solved which is significantly cheaper than solving AREs as in BDF methods. Additional numerical examples can be found in [9].

\section{Conclusions}

Solving Riccati differential equations is a central issue in many applications like, e.g., control design problems. By rewriting the Rosenbrock methods in terms of matrix operations, it turns out that a Sylvester (or Laypunov) equation has to be solved in each time step. The computational cost of solving RDEs by this method is significantly smaller compared with the BDF methods which require the solution of an algebraic Riccati equation in every step. The good stability properties and other nice features of the Rosenbrock methods make these methods a practical alternative to efficiently solve RDEs, especially for the autonomous case which allows an efficient implementation.

Future work will include research on using low-rank approximations to the RDE solution in order to also tackle large-scale problems for which an $\mathcal{O}\left(n^{2}\right)$ memory requirement is prohibitive.

\section{REFERENCES}

[1] H. Abou-Kandil, G. Freiling, V. Ionescu, and G. Jank, Matrix Riccati Equations in Control and Systems Theory. Basel, Switzerland: Birkhäuser, 2003. 1

[2] A. Ichikawa and H. Katayama, "Remarks on the time-varying $H_{\infty}$ Riccati equations," Sys. Cont. Lett., vol. 37(5), pp. 335-345, 1999. 1

[3] E. Davison and M. Maki, "The numerical solution of the matrix Riccati differential equation," IEEE Trans. Automat. Control, vol. 18, pp. 71-73, 1973. 1

[4] C. Kenney and R. Leipnik, "Numerical integration of the differential matrix Riccati equation," IEEE Trans. Automat. Control, vol. AC-30, pp. 962-970, 1985. 1

[5] A. Laub, "Schur techniques for Riccati differential equations," in Feedback Control of Linear and Nonlinear Systems, D. Hinrichsen and A. Isidori, Eds. New York: Sringer-Verlag, 1982, pp. 165-174. 1

[6] E. Arias, V. Hernández, J. Ibáñes, and J. Peinado, "A fixed point-based BDF method for solving Riccati equations," Applied Mathematics and Computation, vol. 188, pp. 1319-1333, 2007. 1

[7] C. Choi and A. Laub, "Efficient matrix-valued algorithms for solving stiff Riccati differential equations," IEEE Trans. Automat. Control, vol. 35, pp. 770-776, 1990. 1, 3

[8] L. Dieci, "Numerical integration of the differential Riccati equation and some related issues," SIAM J. Numer. Anal., vol. 29(3), pp. 781-815, 1992. 1

[9] H. Mena, "Numerical solution of differential riccati equations arising in optimal control problems for parabolic partial differential equations," Ph.D. dissertation, Escuela Politecnica Nacional, 2007. 1, 3, 4, 6

[10] F. Bornemann and P. Deuflhard, Scientific Computing with Ordinary Differential Equations, ser. Text in Applied Mathematics. New York: Springer-Verlag, 2002, vol. 42. 1, 4

[11] E. Hairer and G. Wanner, Solving Ordinary Differential Equations II-Stiff and Differential Algebraic Problems, ser. Springer Series in Computational Mathematics. New York: Springer-Verlag, 2000. 1, 2

[12] P. Lancaster and L. Rodman, The Algebraic Riccati Equation. Oxford: Oxford University Press, 1995. 1, 3

[13] J. Blom, W. Hundsdorfer, E. Spee, and J. Verwer, "A second order Rosenbrock method applied to photochemical dispersion problems," SIAM J. Sci. Comput., vol. 20(4), pp. 1456-1480, 1999. 2

[14] D. A. Voss and A. Q. M. Khaliq, "Parallel Rosenbrock methods for chemical systems," Computers and Chemistry, vol. 25, pp. 101-107, 2001. 2

[15] R. Bartels and G. Stewart, "Solution of the matrix equation $A X+X B=$ $C$ : Algorithm 432," Comm. ACM, vol. 15, pp. 820-826, 1972. 3

[16] G. H. Golub, S. Nash, and C. F. Van Loan, "A Hessenberg-Schur method for the problem $A X+X B=C$," IEEE Trans. Automat. Control, vol. AC-24, pp. 909-913, 1979. 3

[17] P. Benner and H. Mena, "BDF methods for large-scale differential Riccati equations," in Proc. of MTNS 2004, B. D. Moor, B. Motmans, J. Willems, P. V. Dooren, and V. Blondel, Eds., 2004. 3

[18] P. Benner, "Computational methods for linear-quadratic optimization," Supplemento ai Rendiconti del Circolo Matematico di Palermo, Serie II, vol. No. 58, pp. 21-56, 1999. 3

[19] A. Laub, "A Schur method for solving algebraic Riccati equations," IEEE Trans. Automat. Control, vol. AC-24, pp. 913-921, 1979. 3

[20] L. F. Shampine, "Implementation of Rosenbrock Methods," ACM Transactions on Mathematical Software, vol. 8, no. 2, pp. 93-103, 1982. 4

[21] C. Choi and A. Laub, "Constructing Riccati differential equations with known analytic solutions for numerical experiments," IEEE Trans. Automat. Control, vol. 35, pp. 437-439, 1990. 5

[22] J. Abels and P. Benner, "CAREX - a collection of benchmark examples for continuous-time algebraic Riccati equations," SLICOT Working Note 1999-14, 1999, Available from http://www.slicot.org. 5, 6 